\title{
Analysis of Minimum Cost of Supply Chain Emergencies Based on Diffusion Path Processing
}

\author{
Yan Cao $(D$, Tian Tian $(D)$, Wanyu Wei, Liang Huang, and Yujia Wu \\ Mechatronic Engineering, Xi'an Technological University, Xi'an 710021, China \\ Correspondence should be addressed to Yan Cao; caoyan@xatu.edu.cn
}

Received 4 November 2019; Revised 19 March 2020; Accepted 8 June 2020; Published 16 July 2020

Academic Editor: Eric Feulvarch

Copyright (c) 2020 Yan Cao et al. This is an open access article distributed under the Creative Commons Attribution License, which permits unrestricted use, distribution, and reproduction in any medium, provided the original work is properly cited.

\begin{abstract}
In view of the complexity and severity of the impact of supply chain emergencies on enterprise economy, this paper proposes modular processing to improve the design structure matrix (DMS), and the designed clustering algorithm is used to perform cluster analysis of the improved DMS, to predict the possible diffusion path of emergencies, and to establish the critical event diffusion path planning model by designing the critical event diffusion path storage method. As in the case data of a certain type of servo motor of the H Company, after data screening, the diffusion path is classified and stored by analyzing the relationship between each member of the supply chain network. Secondly, the same group of data is put into the method of this paper and other scholars' to calculate the minimum cost of emergency response in time.
\end{abstract}

\section{Introduction}

With the rapid development of economic globalization, the supply chain aims at providing better service, faster meeting demand, lower cost, and closer strategic partnership and continuously strives for excellence, so that the competition of the supply chain is one of the core competitiveness of enterprises [1].

The modern supply chain system is no longer equivalent to the traditional single chain structure, but a complex network structure [2], which integrates a large number of information sharing and supply chain strategies. A supply chain system may consist of multiple suppliers, manufacturers, dealers, and retailers, and the number of upstream and downstream enterprises is increasing for each member [3]. With the increase in the members and the development of lean production, uncertainty, and sudden increase in the supply chain, the supply chain system is vulnerable to the slight changes, and if the relevant emergency mechanism is not put forward in time, it will put the system on the verge of collapse [4]. The outbreak of the SARS virus in 2003 not only affected people's daily life and work but also affected the production and operation of enterprises. Factory shutdown and product shutdown all brought inestimable economic losses to various industries [5]. Therefore, the quality management of the supply chain system is very important to maintain the stable operation of the supply chain system.

Scholars at domestic and abroad have carried out a series of studies on the risk response of emergencies and supply chain. In the analysis of the emergence, development, and evolution of emergencies, Li et al. [6] summarized the four elements that cause emergencies and put forward a chain effect method to describe emergencies in detail. Baghalian et al. [7] considered the uncertainty of the demand side and the supplier side at the same time and established a qualitative analysis model of demand uncertainty, so that the demand became predictable. Pal et al. [8], under the demand of multiple suppliers, single manufacturers, and multiple retailers, established a multiclass product inventory model. Hishamuddin et al. [9] solved the problem of supply demand interruption by establishing a robust stochastic optimization model and a Lagrange model. By using the method of mathematical statistics, the types of emergencies in the supply chain are summarized into eight categories; Sheffi [10], through specific case analysis, concluded that six failure factors, such as failure of supply, failure of transportation, failure of equipment implementation, failure of logistics, failure of information exchange, and failure of demand, 
could lead to the risk of the supply chain, leading to failure. John et al. [11] formulated a dynamic operation and a timely update strategy to control the diffusion by dynamically adjusting the idle time in the recovery period of the sudden event diffusion, so as to save the cost and time of the diffusion control process. According to the characteristics of high uncertainty and sudden and urgent, destructive and diffusive nature of supply chain emergencies, Qin et al. [12] analyzed the main factors that affect the emergency management of the supply chain and formulated the response strategy of supply chain emergencies from three angles of enterprise, government, and consumers. On the basis of the loss assessment model, Dong et al. [13] worked out the loss assessment steps caused by supply chain emergencies and put forward the concept of alliance library, established alliance inventory to respond to supply chain emergencies, and effectively prevented its diffusion impact.

Based on the above research, in order to realize the minimum cost response of the emergency, it is necessary to predict the development path of the emergency, to predict the impact of the early risk in time, and to put forward an effective and timely response mechanism. On the basis of initial DSM, this paper proposes modular processing DSM, which can realize the dynamic integration of each member in the supply chain network. Then, cluster analysis of the member relationship based on module DSM predicts the path of potential diffusion and stores it in the XML structure. Finally, the minimum total cost model of the supply chain network is established. It realizes the effective control of the propagation path and diffusion range of the emergency to minimize the cost of solving the emergency.

\section{Methodology}

\subsection{Analysis of Sudden Event Diffusion Path in Supply Chain}

2.1.1. Modularization of Supply Chain Network. This paper mainly studies the supply chain system composed of three kinds of members, namely, manufacturer, distribution center, and retailer. The DSM module clearly divides the member structures in the supply chain network, creates the condition for the member association relationship analysis in the module, and helps to further analyze and control the relationship members. This paper uses the hierarchical clustering analysis method [14-16]; under the condition of initial DSM, the initial DSM is transformed into reachable by the correlation algorithm DSM, and then the reachable DSM is transformed into a module DSM, which is represented by $n \times n$ class Boolean DSM as shown in Figure 1 .

If there is a reachability relationship among the members, there is an edge connection among the members, which is represented by " 1 ," that is, $n_{i j}=1$, where $i \neq j$; otherwise, $n_{i j}=0$, where $i \neq j$. In the module DSM, the three submodules are independent of each other, and the overlap between submodule 1 and submodule 2 does not affect the independence of the overall modules. When the module to which the member belongs overlaps with other submodules, although the search scope is expanded, it will not spread to the whole world, and it greatly reduces the search time of the associated members and improves the efficiency of emergency response. However, there is an overlap between submodule 1 and submodule 2, especially $n_{\mathrm{u} 3}, n_{\mathrm{v} 2}, n_{\mathrm{v} 3}$, and $n_{\mathrm{u} 1}$ appear in both submodules, which indicates that the changes of these members will affect the two submodules at the same time. In order to reduce the risk degree of the supply chain, it is necessary to strengthen the antirisk ability of these members and minimize the occurrence of emergencies and the expansion of the diffusion range.

A number of methods are used to calculate DSM clustering results, such as original manual calculation, fuzzy clustering analysis, and clustering operation using an intelligent algorithm [17]. Tian et al. [18] used an improved genetic algorithm for intelligent clustering of DSM in the supply chain. In order to carry on the modular management to the emergency task flow, using the advantage of the DSM to carry on the modular management, through the improved genetic algorithm to carry on the cluster analysis to the DSM, they quickly realize the module reorganization.

Based on DSM, this manuscript studies the diffusion path of emergency in the supply chain. Combined with literature [18] data, the DSM hierarchical clustering analysis method is compared with the genetic algorithm in the literature [18]. As shown in Figure 2, when solving the same target, the number of iterations of this algorithm is much smaller than that of the genetic algorithm, which improves the clustering efficiency.

\subsubsection{Classification and Storage of Sudden Event Diffusion} Path. The member position is divided into trigger layer, diffusion layer, and absorption layer according to the level [19]. Different levels of members are arranged and combined into different diffusion paths, and the control methods of different diffusion paths are also different. Emergencies occur randomly in any member. According to the reception of emergencies, the diffusion path can be divided into three categories: type I, the initial emergencies are received by all the members of the absorption layer; type II, the initial emergencies are diffused by the members of the diffusion layer and received by the members of the absorption layer; and type III, the initial emergencies are jointly received by the members of the absorption layer after being absorbed and split by the members of the diffusion layer [20]. Through the diffusion path analysis algorithm, the diffusion path and the corresponding category can be output by inputting the clustering matrix.

Because of the large number of members in the supply chain network, the diffusion path of emergencies becomes very complex. Effective path storage will help to accurately and quickly locate the changed members and control the diffusion path. XML (extensible markup language) is used to store diffusion information, such as in Figure 3, which is the XML storage structure of the emergency diffusion path.

\subsection{Minimum Total Cost Model for Supply Chain Emergencies}

\subsubsection{Assumed Condition}

(1) In the face of emergencies, time will directly affect the scope and effect of emergencies, so the time will 


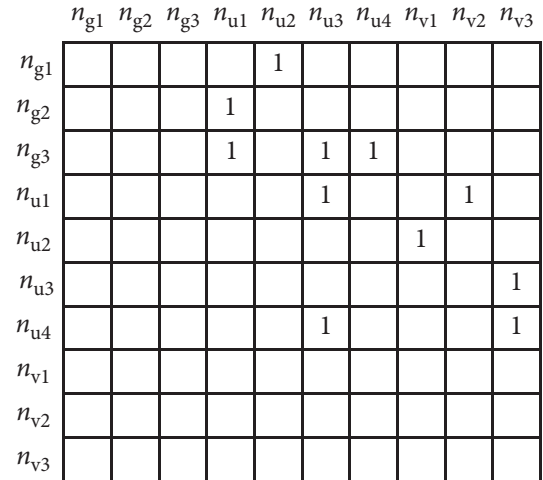

Initial DSM

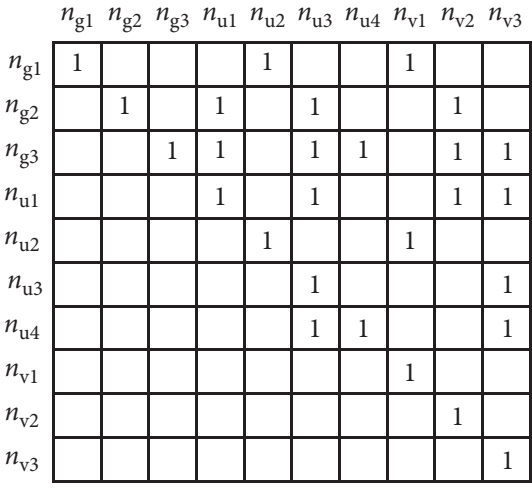

Reachable DSM

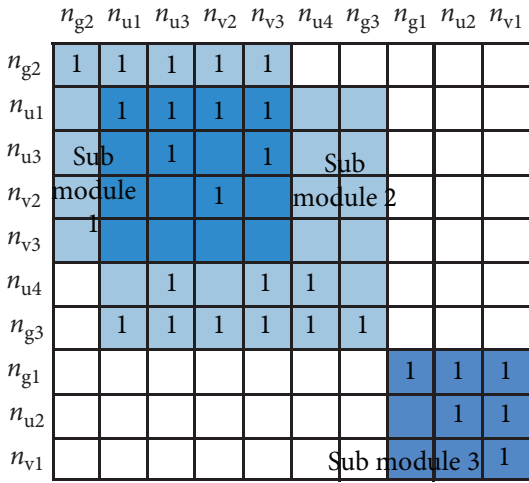

Module DSM

Figure 1: Boolean DSM.

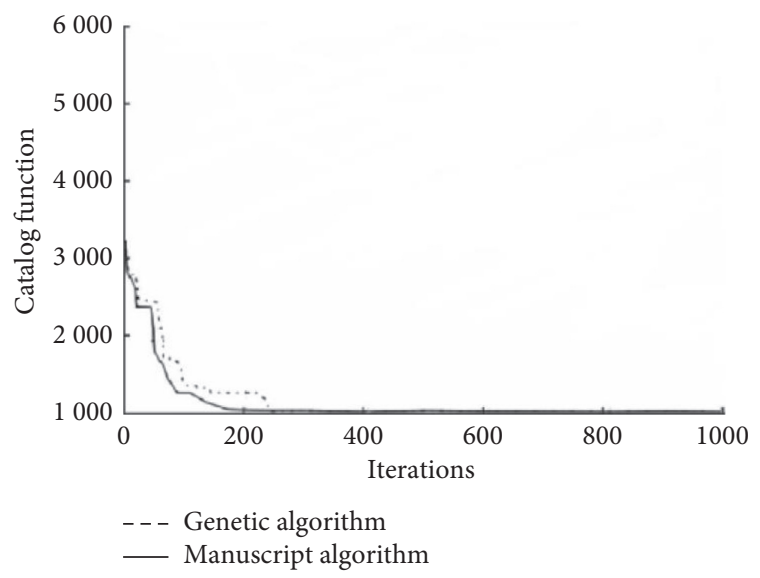

FIGURE 2: Manuscript algorithm parallels to the genetic algorithm.

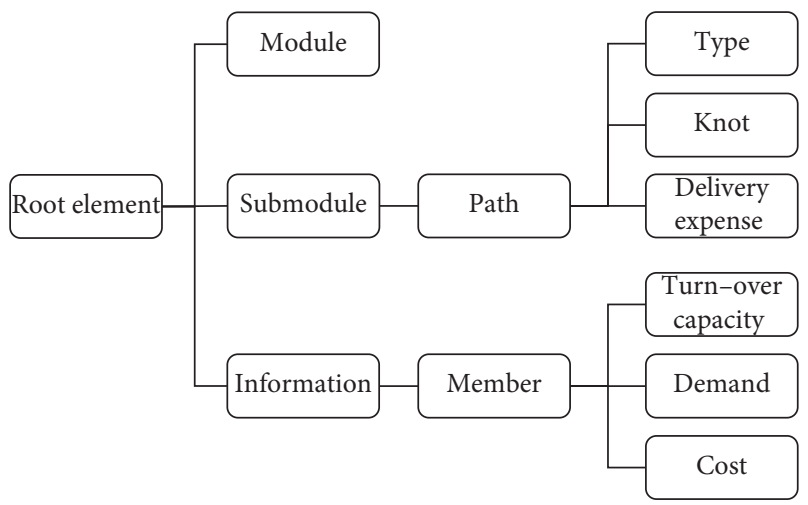

Figure 3: XML storage structure for the emergency diffusion path.

be considered in the model establishment. When the emergency among the members occurs at $T_{n}$ time, the supply chain system will return to a stable status in the future at a limited time interval, that is, the duration time of the emergency is $t_{\mathrm{d} n}$, and meet the requirements $T_{n}+t_{\mathrm{d} n} \leq 1$.

(2) In this paper, the manufacturers only produce one kind of products, and the types of products circulating in the supply chain network are the same.
(3) The number of intervals in the recovery time window is decided by the members of the supply chain, and the number of intervals in the recovery time window is the same for each member.

(4) In this paper, the members of the fast recovery strategy agree with each other.

\subsubsection{Model Construction}

(1) Manufacturer cost:

Production cost:

production cost $=\sum_{k=1}^{G} p_{\mathrm{g} k} P_{\mathrm{g} k}^{\prime}$

Holding cost:

$$
\text { holding cost }=t_{\mathrm{d} n} \times \sum_{k=1}^{G} H_{\mathrm{g} k}\left(P_{\mathrm{g} k}^{\prime}+S C_{\mathrm{g} k}\right) \text {. }
$$

Transportation cost:

$$
\text { transportation cost }=\sum_{k^{\prime}=1}^{\mathrm{U}} \sum_{k=1}^{G} T_{\mathrm{g} k_{-} \mathrm{u} k^{\prime}} T Q_{\mathrm{g} k_{-} \mathrm{u} k^{\prime}}^{\prime}
$$

(2) Distribution center cost:

Operating cost_DC:

$$
\text { operating cost_DC }=t_{\mathrm{d} n} \times \sum_{k^{\prime}=1}^{\mathrm{U}} O C_{\mathrm{uk}} \text {. }
$$

Handling cost cost_DC:

handing cost_DC $=t_{\mathrm{d} n} \times \sum_{k^{\prime}=1}^{\mathrm{U}} \sum_{k=1}^{G} H_{\mathrm{u} k^{\prime}}\left(T Q_{\mathrm{g} k_{-} \mathrm{u} k^{\prime}}^{\prime}+S C_{\mathrm{u} k^{\prime}}\right)$.

Transportation cost_DC: 
transportation cost_DC $=\sum_{k^{\prime \prime}=1}^{\mathrm{V}} \sum_{k^{\prime}=1}^{\mathrm{U}} T_{\mathrm{u} k^{\prime} \_\mathrm{v} k^{\prime \prime}} T Q_{\mathrm{u} k^{\prime} \_\mathrm{v} k}^{\prime} \cdot$

(3) Retail cost:

Holding cost_R :

$$
\text { holding cost_R }=\left[t_{\mathrm{d} n} \times \sum_{k^{\prime \prime}=1}^{\mathrm{V}} H_{\mathrm{v} k^{\prime \prime}}\left(D_{\mathrm{v} k^{\prime \prime}}+S C_{\mathrm{v} k^{\prime \prime}}\right)\right] \text {. }
$$

The formula (1) is added to formula (7), and the total target cost is minimized.

Minimum total target cost $=$ manufacturer $\cos t+$ dis tribution center cost + retail cost:

$\min$ total cost $=\min \left(\begin{array}{c}\sum_{k=1}^{G} p_{\mathrm{g} k} P_{\mathrm{g} k}^{\prime}+\left[t_{\mathrm{d} n} \times \sum_{k=1}^{G} H_{\mathrm{g} k}\left(P_{\mathrm{g} k}^{\prime}+S C_{\mathrm{g} k}\right)\right]+\sum_{k^{\prime}=1}^{\mathrm{U}} \sum_{k=1}^{G} T_{\mathrm{g} k_{-} \mathrm{u} k^{\prime}} T Q_{\mathrm{g} k_{-} \mathrm{u} k^{\prime}}^{\prime}+\sum_{k^{\prime \prime}=1}^{\mathrm{V}} \sum_{k^{\prime}=1}^{\mathrm{U}} T_{\mathrm{u} k^{\prime}-\mathrm{v} k^{\prime \prime}} T Q_{\mathrm{u} k^{\prime}-\mathrm{v} k^{\prime \prime}}^{\prime} \\ +\left[t_{\mathrm{d} n} \times \sum_{k^{\prime}=1}^{\mathrm{U}} O C_{\mathrm{u} k^{\prime}}\right]+\left[t_{\mathrm{d} n} \times \sum_{k^{\prime}=1}^{\mathrm{U}} \sum_{k=1}^{G} H_{\mathrm{u} k^{\prime}}\left(T Q_{\mathrm{g} k_{-} \mathrm{u} k^{\prime}}^{\prime}+S C_{\mathrm{u} k^{\prime}}\right)\right]+\left[t_{\mathrm{d} n} \times \sum_{k^{\prime \prime}=1}^{\mathrm{V}} H_{\mathrm{v} k^{\prime \prime}}\left(D_{\mathrm{v} k^{\prime \prime}}^{\prime}+S C_{\mathrm{v} k^{\prime \prime}}\right)\right]\end{array}\right)$.

\subsubsection{Constraint Condition}

(1) The sum of the traffic from the manufacturer $g$ to the distribution center $u$ is less than or equal to the sum of the maximum capacity and reserve capacity of the manufacturer g:

$\sum_{k^{\prime}=1}^{\mathrm{U}} \sum_{k=1}^{G} T Q_{\mathrm{g} k_{-} \mathrm{u} k}^{\prime} \leq \sum_{k=1}^{G}\left(P C_{\mathrm{g} k}+S C_{\mathrm{g} k}\right)$.

(2) The sum of the traffic from the manufacturer $g$ to the distribution center $u$ is greater than or equal to the maximum management capacity of the distribution center $u$ :

$$
\sum_{k^{\prime}=1}^{\mathrm{U}} \sum_{k=1}^{G}\left(T Q_{\mathrm{g} k_{-} \mathrm{u} k^{\prime}}^{\prime}+S C_{\mathrm{u} k^{\prime}}\right) \geq \sum_{k^{\prime}=1}^{\mathrm{U}} H C_{\mathrm{u} k^{\prime}}
$$

(3) The sum of the traffic from the distribution center $u$ to the retailer $\mathrm{v}$ is less than or equal to the maximum management ability of the distribution center:

$$
\sum_{k^{\prime \prime}=1}^{\mathrm{V}} \sum_{k^{\prime}=1}^{\mathrm{U}} T Q_{\mathrm{u} k^{\prime}-\mathrm{v} k^{\prime \prime}}^{\prime} \leq \sum_{k^{\prime}=1}^{\mathrm{U}}\left(H C_{\mathrm{u} k^{\prime}}+S C_{\mathrm{u} k^{\prime}}\right) \text {. }
$$

(4) The sum of the traffic from the distribution center $u$ to the retailer $\mathrm{v}$ is greater than or equal to the demand of the retailer $\mathrm{v}$ :

$$
\sum_{k^{\prime \prime}=1}^{\mathrm{V}} \sum_{k^{\prime}=1}^{\mathrm{U}} T Q_{\mathrm{u} k^{\prime}-\mathrm{v} k^{\prime \prime}}^{\prime} \geq \sum_{k^{\prime \prime}=1}^{\mathrm{V}}\left(D_{\mathrm{v} k^{\prime \prime}}^{\prime \prime}+S C_{\mathrm{v} k^{\prime \prime}}\right) \text {. }
$$

(5) After the emergency occurs and continues $t_{\mathrm{d} n}$, the manufacturer g output is less than or equal to the manufacturer $g$ maximum production capacity in the ideal environment:

$$
\sum_{k=1}^{G} P_{\mathrm{g} k}^{\prime} \leq \sum_{k=1}^{G} P C_{\mathrm{g} k}
$$

(6) After the emergency occurs and lasts $t_{\mathrm{d} n}$, the capacity of the distribution center $u$ is less than or equal to the maximum management ability of the distribution center $u$ :

$$
\sum_{k^{\prime}=1}^{\mathrm{U}} H C_{\mathrm{u} k^{\prime}}^{\prime} \leq \sum_{k^{\prime}=1}^{\mathrm{U}} H C_{\mathrm{u} k^{\prime}}
$$

(7) All variables are greater than 0 :

$$
\begin{aligned}
& P_{\mathrm{g} k}^{\prime}, D_{\mathrm{v} k^{\prime \prime}}^{\prime}, H C_{\mathrm{u} k^{\prime}}^{\prime}, H_{\mathrm{g} k}, H_{\mathrm{u} k^{\prime}}, H_{\mathrm{v} k_{\prime \prime}}, T_{1 \mathrm{~g} k_{-} \mathrm{u} k^{\prime}}, T_{2 \mathrm{u} k^{\prime}-\mathrm{v} k^{\prime \prime}}, \\
& T Q_{\mathrm{g} k_{-} \mathrm{u} k^{\prime}}^{\prime}, T Q_{\mathrm{u} k^{\prime} \_\mathrm{v} k^{\prime \prime}}^{\prime}, O C_{\mathrm{u} k^{\prime}}, P C_{\mathrm{g} k}, H C_{\mathrm{u} k^{\prime}} \geq 0, \forall k, k^{\prime}, k^{\prime \prime} .
\end{aligned}
$$

The variable setting in formulas (1)-(15) is shown in Table 1.

\section{Experiment and Analysis}

3.1. Analysis of Association Relationship between Emergency Members in Supply Chain. Based on the background of the $\mathrm{H}$ Company, this paper expounds the analysis process of real data and the display of the results on its transportation management platform and realizes the method of this paper. In the process of data screening, the research product is located in a certain type of servo motor in Jiangsu and Anhui provinces of the $\mathrm{H}$ Company, and the specific data information is extracted and analyzed for a certain customer group of the product in a certain period of time.

The two manufacturing plants in Jiangsu and Anhui are located in Suzhou, and a certain type of servo motor is mainly produced in Suzhou Factory 1. In order to alleviate the inventory pressure and shipping demand of Factory 1, 
TAвLE 1: Variable representation table.

\begin{tabular}{|c|c|}
\hline Variable & Variable meaning \\
\hline $\mathrm{g}$ & Manufacturer \\
\hline u & Distribution center \\
\hline $\mathrm{v}$ & Retailers \\
\hline G & Number of manufacturers \\
\hline$U$ & Number of distribution centers \\
\hline$V$ & Number of retailers \\
\hline$T_{n}$ & Start time of the risk of member $n$ \\
\hline$t_{\mathrm{d} n}$ & Duration of the risk of member $n$ \\
\hline$p_{\mathrm{g} k}$ & Unit production cost of manufacturer $g k$ \\
\hline$P_{\mathrm{g} k}^{\prime \prime \prime}$ & Production of manufacturer $g k$ after the forecast \\
\hline$P C_{\mathrm{g} k}$ & The maximum productivity of manufacturer $g k$ per unit time in an ideal environment \\
\hline$H_{\mathrm{g} k}$ & Manufacturer $g k$ unit time unit inventory maintenance cost \\
\hline$H_{\mathrm{u} k^{\prime}}^{\delta^{\prime \prime}}$ & Distribution center $\mathrm{u} k^{\prime}$ unit time unit management cost \\
\hline$H_{\mathrm{v} k^{\prime \prime}}$ & Retail $v k^{\prime \prime}$ unit time unit inventory cost \\
\hline$H C_{\mathrm{u} k^{\prime}}$ & The maximum management capacity of the distribution center $u k^{\prime}$ \\
\hline$H C_{\mathrm{u} k^{\prime}}^{\prime}$ & Capacity of distribution center $u k^{\prime}$ after forecast \\
\hline$D_{\mathrm{v} k^{\prime \prime}}^{\prime \prime}$ & Retail $v k^{\prime \prime}$ demand after forecast \\
\hline$S C_{n}$ & Standby capacity of member $n$ \\
\hline$T_{\mathrm{g} k \_\mathrm{u} k^{\prime}}$ & Unit transportation costs from manufacturer $g k$ to distribution center $\mathrm{u} k^{\prime}$ \\
\hline$T_{\mathrm{u} k^{\prime}-\mathrm{v} k^{\prime \prime}}^{\mathrm{g}-n_{0}}$ & Unit transportation cost from distribution center $\mathrm{u} k^{\prime}$ to retail $\mathrm{v} k^{\prime \prime}$ \\
\hline$O C_{\mathrm{u} k^{\prime}}^{-v \kappa}$ & Unit time of operating cost of distribution center $u k^{\prime}$ \\
\hline$T Q_{\mathrm{g} k \_\mathrm{u} k^{\prime}}$ & The manufacturer's $g k$ traffic to the distribution center $\mathrm{u} k^{\prime}$ after forecasting \\
\hline$T Q_{\mathrm{u} k^{\prime} \_v k^{\prime \prime}}^{\prime \prime}$ & The volume of traffic from the distribution center $u k^{\prime}$ to the retail $v k^{\prime \prime}$ after forecasting \\
\hline
\end{tabular}

some motors will be shipped to Suzhou Factory 2 in batches, so both factories have a certain type of servo motor. The products of the $\mathrm{H}$ Company are mainly distributed to different customers through agents, only for big customers to take direct sales. Therefore, a certain type of servo motor is mainly sent to three agents in Jiangsu and Anhui provinces, and then the products are sold to customers by agents. The transportation relationship is shown in Figure 4.

The algorithm is written by the Python language and implemented by Jupyter. In the early stage, the initial design structure matrix and unit matrix are mainly input. The output content mainly has the reachability matrix and the number of iterations. The transportation clustering design mechanism matrix $C$ of a certain type of servo motor in Jiangsu and Anhui areas of the H Company is obtained as shown in Figure 5.

After the algorithm runs, the clear clustering results can be obtained, and the two modules can be obtained from Figure 4 , and there is overlap between them. Suzhou factory 1 , agent 1 , agent 2 , and customers 2, 4, 5, and 6 aggregate into a module, Suzhou factory 2, agents 2 and 3, and customers 1, 2, 3, 4, and 6 aggregate into a module, and agent 2 and customers 2, 3, 4, and 6 are overlapping parts of the two modules, so the overlapping part members should pay attention to under normal operation; once the overlapping part of the member has an emergency, the two modules can be disturbed, and all members of the whole system can change as a result of emergencies.

The association information of the members in the two modules is calculated by the algorithm in Figure 4, and five diffusion paths are obtained. From the diffusion path, we can know what kind of association relationship exists between the members and the complexity among the associated members. According to the characteristics of the membership in the path, the path is classified and the path information is stored by XML. The storage content includes not only the basic member name, but also the member number, name, type, association level, contact information, receiving address, receiving quantity, received quantity, uncollected quantity, unit transportation cost, transfer capacity of distribution center, customer demand, and inventory cost. The specific classification and storage are shown in Table 2.

Taking the type III transportation path in Table 2 as an example, the H Company uses XML to store type III path members and their information in the database of transportation management platform and adds the risk prevention module to the original transportation management platform. In this module, according to the idea of association relationship analysis, the associated members can be found by searching the member number, and the information and relationship diagram of the associated members can be obtained. When there is the possibility of abnormal occurrence in Suzhou Factory 2, the influence on its related members can be prevented or even reduced in advance. The function realization effect diagram is shown in Figure 6.

3.2. Example Simulation of Supply Chain Emergency. This paper is based on the background of the collection of the actual data members, the transportation cost of the member units, the unit inventory maintenance cost, the member output, the member unit time operation cost, and so on in the $\mathrm{H}$ Company; the minimum total cost model is used to simulate the emergency response in time, and the data in this paper are introduced into other research methods to compare the results, which shows the superiority of this proposed model and method. 


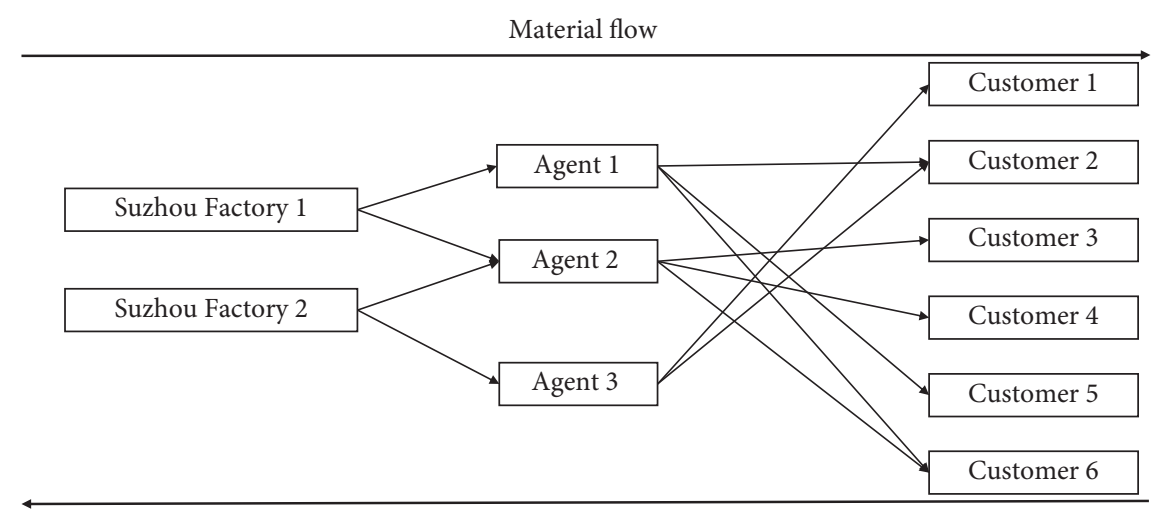

Information flow

Figure 4: Transportation network diagram of a type of servo motor in the H Company.

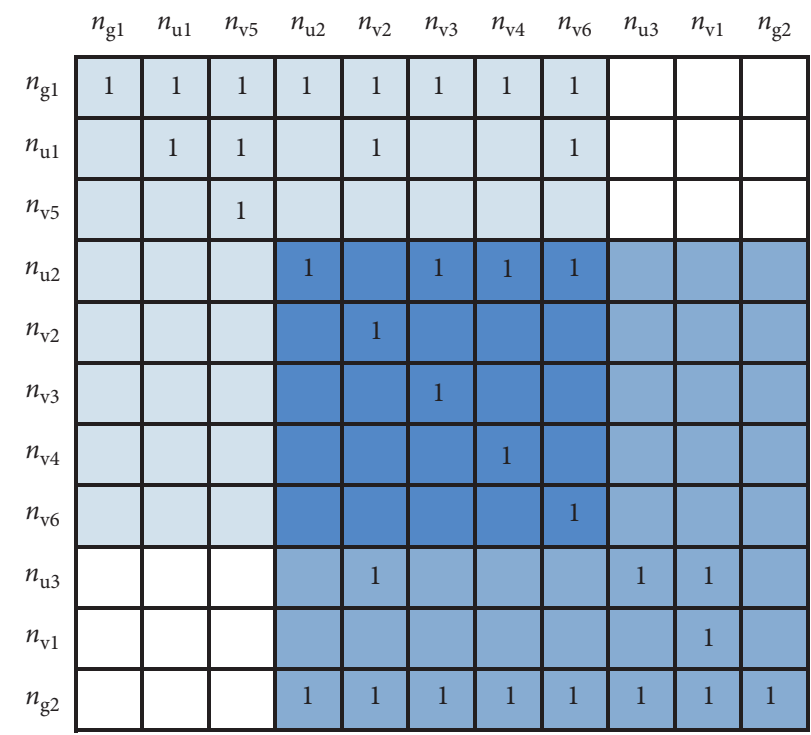

FIgURE 5: A type of servo motor transportation cluster design structure matrix $C$ in the H Company.

TABLE 2: Classification and storage table of the transportation path of a certain type of servo motor in the H Company.

Type I

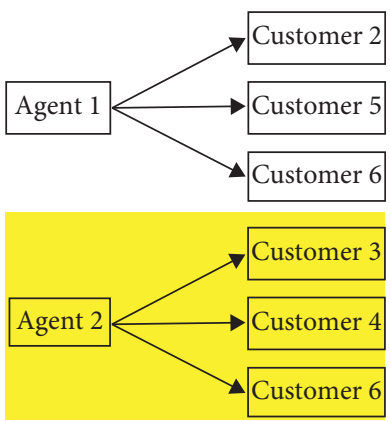

Type I

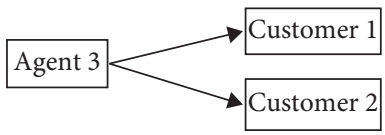


TABle 2: Continued.

Type Correlation diagram

Type III

Type III
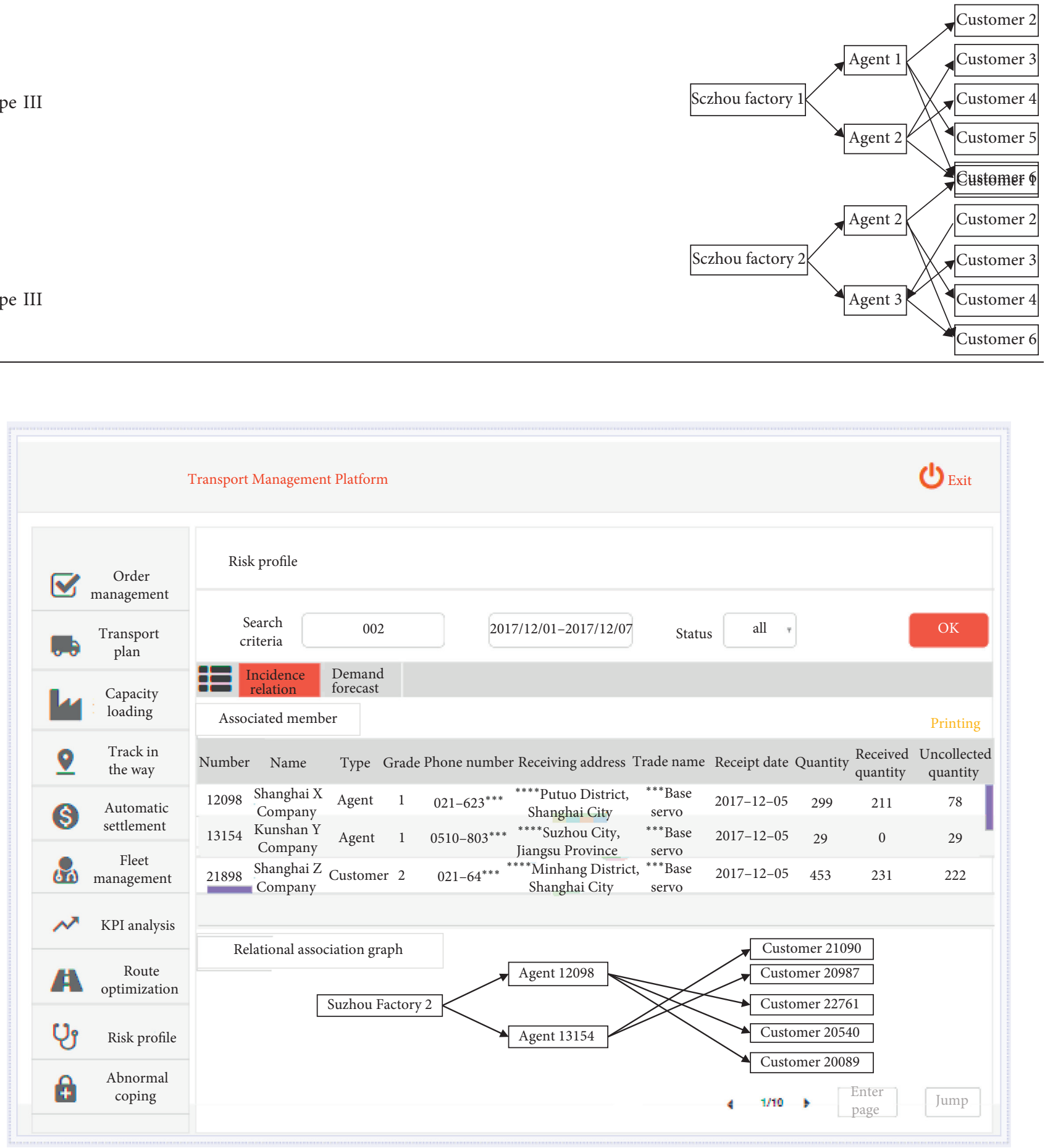

Figure 6: Implementation diagram of association relationship prediction.

3.2.1. Calculation of the Minimum Total Cost. All the data, such as members number, members unit transportation cost, members unit time unit inventory maintenance cost, members output, and members unit time operation cost, are brought into the minimum total cost module formula (8) as input, and the output is the transportation quantity among the members. After programming with the Python language, it is implemented in Jupyter. The results are represented in Figure 7, and the figure shows the quantity of the transported goods, and it can be seen that when emergencies occur in the supply chain, not only the demand quantity changes, but also the transportation path and the transportation quantity 


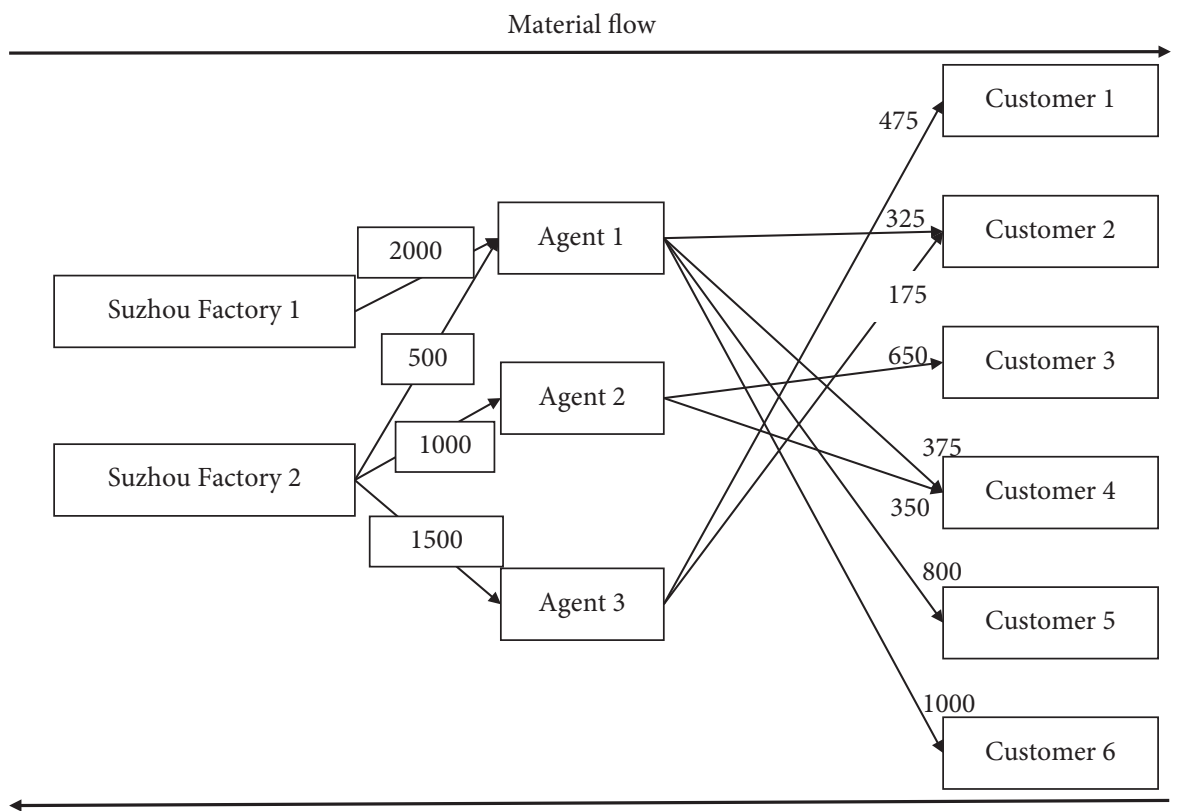

Information flow

Figure 7: Visual representation of the calculation results of the model.

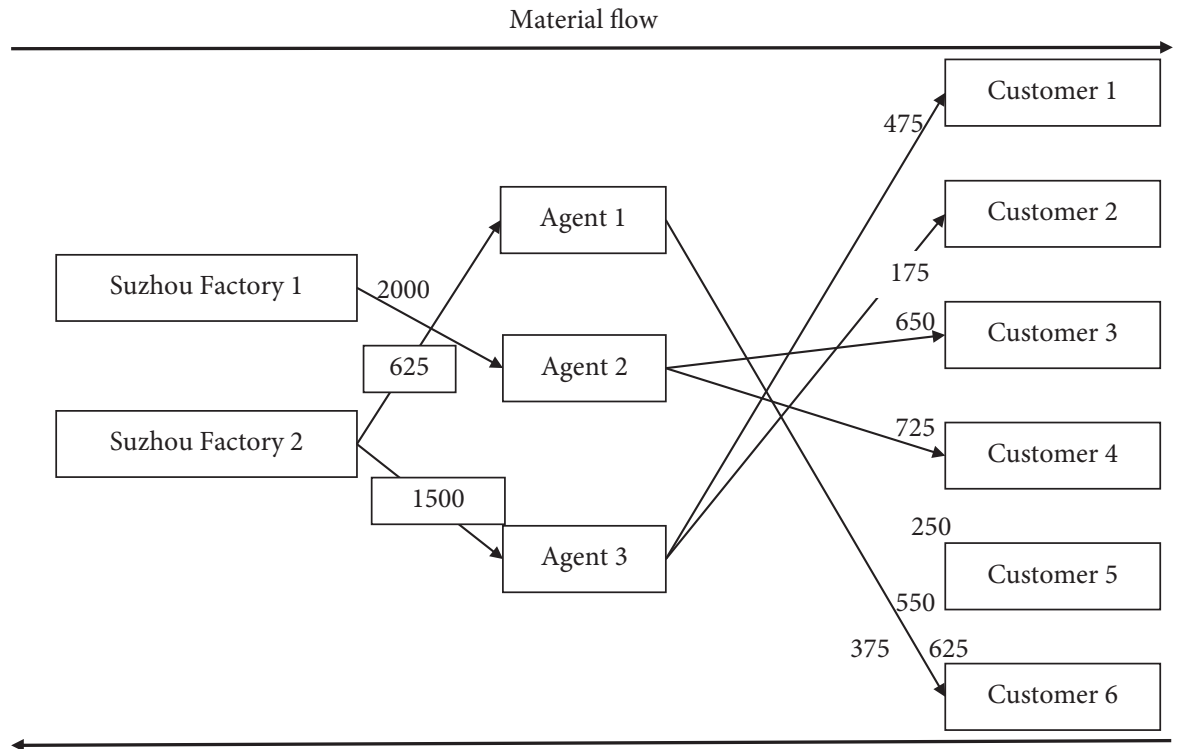

Information flow

Figure 8: Visual representation of the calculation results of the Paul model.

will change, and the adjusted minimum total cost is 1037624.5 RMB.

\subsubsection{Minimum Total Cost Calculation of the Paul Method.} Bringing all the data into the Paul cost model to get the member relationship path and replanning as shown in Figure 8, the calculated minimum total cost is 2069131.5.

In order to study the advantages of the minimum total cost model proposed in this paper, we choose multiple sets of data into the minimum total cost model of this paper and
Paul method, respectively, and get the cost contrast diagram as shown in Figure 9.

In Figure 8, 10 sets of experimental data are selected, and the transverse coordinates represent the number of experiments, and the longitudinal coordinates represent the minimum total cost under different experimental data. From Figure 8 , it can be seen that

(1) In the first 5 trial data, the unit time inventory maintenance cost of the member remains unchanged, and the other individual data will be changed. The calculation result of Paul's model is 


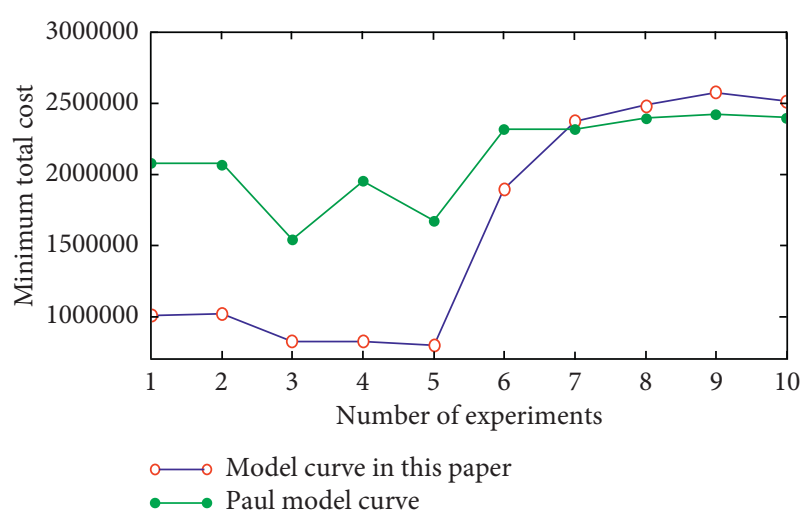

FIgURE 9: Comparison of the minimum total cost.

almost twice that of the minimum total cost model in this paper. And, the result curve of this paper model is more stable, which is because this paper puts the dead storage into the model calculation, when the unexpected situation in the supply chain causes an influence on the member supply, and the dead storage has the buffer effect and reduces the sudden event to the supply chain huge fluctuation.

(2) From the 6th experiment, the cost calculation results of the minimum total cost model in this paper continue to rise and exceed the cost calculation results of Paul. This is due to the fact that, from the 6th experiment, the unit time inventory maintenance cost of members has gradually increased, while the other data have remained basically unchanged. In the first five trials, the unit time inventory maintenance cost was $1 / 3$ of the member unit transportation cost. In the subsequent experiment, the cost of unit time inventory maintenance increases gradually until it exceeds the transportation cost of members. At this time, we find that the cost calculation advantage of the Paul method is slowly reflected.

To sum up, this paper takes into consideration the calculation results of the minimum cost model of safety stock, which is far superior to the minimum cost model of Paul.

\section{Conclusion}

As we can see from the impact of the global spread of the COVID-19 on all aspects of society, in the era of economic globalization, the links between the various components of the supply chain system are becoming more and more close and complex, and problems in any branch will have an impact on the whole supply chain system and increase the difficulty of predicting the direction of the problems in the end branch of the supply chain system. Therefore, in order to reduce the loss caused by emergencies as much as possible, scholars at home and abroad have discussed many aspects. Xialin $\mathrm{Hu}$ builds the supply chain platform of emergency resources with the help of big data technology and controls all kinds of elements of the supply chain through big data technology to ensure the timely supply and maximum utilization of resources; Thomas et al. put forward a dynamic simulation method, through the study of different interruption modes of emergencies, to provide some insights into the parameter setting of designing an emergency procurement strategy; Gholamreza Bodaghi et al. developed different prediction models in different stages through historical data and model verification through practical cases. These studies take the data in the supply chain system as the research object, through the empirical data research, and establish the corresponding analysis model.

In this paper, the propagation path of the supply chain system is analyzed, the structure matrix is designed, and the maximum diffusion range and diffusion path can be predicted under the condition of unknown causal association by member clustering. This method has a clear mathematical mechanism, obtains clear and intuitive results, improves the clustering process, greatly reduces the number of iterations, improves the operation efficiency, and then establishes the minimum total cost model. As a case study, we can see from the experimental results that the minimum cost of this method has obvious advantages, which can not only determine the necessity of the existence of reserve inventory, but also reduce the cost of inventory maintenance. In connection with real life, it can be found that Amazon, JD.com, and other large e-commerce enterprises have chosen the way to build local warehouses to reduce costs, precisely because the inventory cost of a local warehouse has a great advantage over the transportation cost, which again verifies the feasibility of the minimum total cost model established in this paper.

\section{Data Availability}

All data included in this study are available upon request by contact with the corresponding author.

\section{Conflicts of Interest}

The authors declare that they have no conflicts of interest.

\section{Acknowledgments}

This research was funded by the National Natural Science Foundation of China (NSFC) under grant no. 51705392 and the Project of 2018 Shaanxi Province Key Research and Development under grant no. 2018ZDXM-GY-077.

\section{References}

[1] P. Samaranayake, "A conceptual framework for supply chain management: a structural integration," Supply Chain Management, vol. 10, no. 1, pp. 47-59, 2005.

[2] V. G. Whitelock, "Alignment between green supply chain management strategy and business strategy," International Journal of Procurement Management, vol. 5, no. 4, pp. 430451, 2012.

[3] Y. K. Dwivedi, M. A. Shareef, B. Mukerji, N. P. Rana, and K. Kapoor, "Involvement in emergency supply chain for disaster management: a cognitive dissonance perspective," International Journal of Production Research, vol. 56, no. 21, pp. 1-16, 2017. 
[4] B. Shen and Q. Li, "Market disruptions in supply chains: a review of operational models," International Transactions in Operational Research, vol. 24, no. 4, 2017.

[5] S. K. Paul, R. Sarker, and D. Essam, "A quantitative model for disruption mitigation in a supply chain," European Journal of Operational Research, vol. 257, no. 3, pp. 881-895, 2017.

[6] M. Li, J. Chen, T. Chen et al., "Probability model of event chain for emergencies," Journal of Tsinghua University, vol. 50, no. 8, pp. 1173-1177, 2010.

[7] A. Baghalian, S. Rezapour, and R. Z. Farahani, "Robust supply chain network design with service level against disruptions and demand uncertainties: a real-life case," European Journal of Operational Research, vol. 227, no. 1, pp. 199-215, 2013.

[8] B. Pal, S. S. Sana, and K. Chaudhuri, "A three layer multi-item production-inventory model for multiple suppliers and retailers," Economic Modelling, vol. 29, no. 6, pp. 2704-2710, 2012.

[9] H. Hishamuddin, R. A. Sarker, and D. Essam, "A recovery model for a two-echelon serial supply chain with consideration of transportation disruption," Computers \& Industrial Engineering, vol. 64, no. 2, pp. 552-561, 2013.

[10] Y. Sheffi, "Supply chain management under the threat of international terrorism," The International Journal of Logistics Management, vol. 12, no. 2, pp. 1-11, 2003.

[11] J. B. John 3rd., D. D. Eisenstein, L. K. Platzman, R. J. Clark, and D. W. Williamson, "Building a self-organizing urban bus route," in Proceedings of the Sixth International Conference on Self-Adaptive and Self-Organizing Systems Workshops, pp. 66-70, IEEE, Lyon, France, September 2012.

[12] Y. Qin, X. Cao, and Q. Song, "Strategies to improve the ability of supply chain to deal with Emergency Management," Enterprise Economy, vol. 2, pp. 65-68, 2013.

[13] J. Dong, J. Ji, and Q. Sun, "Research on loss evaluation of supply chain emergencies," Statistics and Decision-Making, vol. 9, pp. 173-175, 2011.

[14] V. Mani, R. Agarwal, A. Gunasekaran, T. Papadopoulos, R. Dubey, and S. J. Childe, "Social sustainability in the supply chain: construct development and measurement validation," Ecological Indicators, vol. 71, pp. 270-279, 2016.

[15] Z. Babai, J. E. Boylan, S. Kolassa et al., "Supply chain forecasting: theory, practice, their gap and the futureauthor-name: syntetos, aris a," European Journal of Operational Research, vol. 252, no. 1, pp. 1-26, 2016.

[16] P. Kouvelis and W. Zhao, "Supply chain contract design under financial constraints and bankruptcy costs," Management Science, vol. 62, no. 8, pp. 2341-2357, 2016.

[17] W. Jiang, E. H.-M. Sha, Q. Zhuge, and L. Wu, "Efficient assignment algorithms to minimize operation cost for supply chain networks in agile manufacturing," Computers \& Industrial Engineering, vol. 108, pp. 225-239, 2017.

[18] J. Tian, L. Li, J. Bai, and G. Feng, "Modular design of emergency task executive processes based on DSM," Chinese Journal of Management Science, vol. 22, no. 8, pp. 100-107, 2014.

[19] L. V. Snyder, Z. Atan, P. Peng, Y. Rong, A. J. Schmitt, and B. Sinsoysal, "OR/MS models for supply chain disruptions: a review," IIE Transactions, vol. 48, no. 2, pp. 89-109, 2016.

[20] Z. Zhang, "Research on the stability of supply chain based on planned output random and information asymmetry," Logistics Engineering and Management, vol. 40, no. 9, pp. 92-95, 2018. 